\title{
CRYPTOSPORIDIOSIS: AN EMERGING ENTERIC DISEASE IN HIV-INFECTED PATIENTS, BARAK VALLEY, ASSAM, INDIA
}

\author{
GULZAR HUSSAIN ${ }^{1,2}$, SHUBHADEEP ROYCHOUDHURY², BABY SINGHA ${ }^{*}$ \\ ${ }^{1}$ Molecular Parasitology Laboratory, Department of Zoology, G. C. College, Silchar, India, ${ }^{2}$ Department of Life Science and Bioinformatics, \\ Assam University, Silchar, India \\ Email: babysingha@gmail.com
}

Received: 10 Mar 2016 Revised and Accepted: 22 Jul 2016

ABSTRACT

Objective: Enteric parasites are a major cause of diarrhoea in HIV-infected people. The present study was undertaken to detect enteric parasites in HIV-infected patients with diarrhoea at different levels of immunity.

Methods: The study was carried out at the ART centre Silchar Medical College and Hospital, Assam, India, between March 2013 and March 2014 among consecutively enrolled 127 HIV-infected patients presenting with diarrhoea. Stool samples were collected and examined for enteric parasites by microscopy and special staining methods. CD4 cell counts were estimated using the rapid serological tests using Combs Aids, Pareekshak® HIV $1 / 2$ Triline card test and SD Bioline anti-HIV $1 / 2$ test kits as per protocol provided by the supplier.

Results: A total of 127 stool samples was collected from the HIV patients attending the antiretroviral therapy (ART) center at Silchar Medical College Hospital in Assam and were isolated for Cryptosporidium oocysts during the one year period from March 2013 and March 2014 . The stool samples were examined by the help of modified Ziehl-Neelsen technique. It was seen that out of 127 samples, 25 were Cryptosporidium oocysts positive (19.68\%), which is the higher than the prevalence range reported from the Southern Assam of India.

Conclusion: Cryptosporidium infections were detected in 19. 68 percent HIV-infected patients and low CD4 counts were significantly associated with infection. Detection of aetiologic pathogens might help clinicians decide appropriate management strategies.

Keywords: Diarrhoea, HIV, Cryptosporidium spp

(C) 2016 The Authors. Published by Innovare Academic Sciences Pvt Ltd. This is an open access article under the CC BY license (http://creativecommons. org/licenses/by/4. 0/) DOI: http://dx.doi.org/10.22159/ijpps.2016.v8i9.11625

Cryptosporidium spp. causes severe, life-threatening diarrhoea in untreated HIV-infected patients. Until the advent of highly active anti-retroviral therapy (HAART), this was a relatively common opportunistic infection even in developed countries $[1,4]$. In India, there have been reports from the mid-1990s on the prevalence of cryptosporidiosis from different parts of the country, ranging from 8.5 [5] to $81 \%$ [6] with a very high incidence reported from the northeastern states $[6,7]$.

Cryptosporidiosis is a parasitic disease caused by the Cryptosporidium, a protozoan parasite belonging to the phylum Apicomplexa. Cryptosporidium was discovered by Tyzzer in 1907 from the gastric glands of infected mice, and it gradually emerged as an increasingly recognized public health threat $[8,9]$. It has been identified in watery diarrhoea in patients with HIV and other immune-compromised patients and in large human outbreaks of diarrhoea in both developed and developing parts around the world $[10,11]$. It affects the intestines of mammals. It spreads through the fecal-oral route and contaminated water. In immunecompromised persons, such as AIDS patients, the symptoms are severe and often fatal $[9,11]$.

The emergence and pandemic spread of AIDS constitute the greatest challenge to public health in modern times. The first case of AIDS in India reported in 1986 from Chennai. India is now the country with the second largest population of HIV-infected individuals [12].

Cryptosporidium is a genus of protozoan parasites with species that infect fish, amphibians, reptiles, birds and mammals. In HIV-positive patients suffering from diarrhoea it is the most commonly isolated parasite. Oocysts are transmitted from an infected host to susceptible hosts. Transmission can be human-to-human, animal-toanimal, human-to-animal, and animal-to-human, waterborne, foodborne and possibly airborne. In the case of HIV-positive patients, generally, CD4 T cell numbers or function decreases, thereby increasing the severity of the infection. Infections can become chronic and life is threatening with frequent voluminous watery stools leading to dehydration [13].

The objective of this study is to examine the prevalence of Cryptosporidium spp. occurring in hospital-based patients with diarrhoeal symptoms during the period of one year from March 2013 to March 2014.

The study was approved by the Ethical Committee of the institution (Ref no. GCC/9440). All study participants had given written consent before enrollment into the study. Parents/guardians provided consent on behalf of all infant participants. Fecal samples were collected from a total of 127 HIV-positive patients attending the ART center of the Silchar Medical College and Hospital, Assam. Cryptosporidium oocysts were identified by the modified ZeihlNeelsen technique [14]. The Cryptosporidium-positive samples were kept on $-20^{\circ} \mathrm{C}$ to be used for DNA extraction.

Isolation of Cryptosporidium species was carried out by modified Ziehl-Neelsen staining technique according to Potters and Esbroeck (2010) with slight modifications as given below:

Preparation of smear for staining involved applying a small sample to the center of a carefully cleaned glass slide. The smear was allowed to dry completely and then the smear was fixed with methanol. The smear was heat fixed, and air dried at $80{ }^{\circ} \mathrm{C}$ for 15 min. A slide was placed on an air dried and heat fixed smear on a suitable staining device; then the slide was flooded with Kinyoun's carbolfuchsin reagent and allowed to stain for $15 \mathrm{~min}$. The slide was washed using a gentle and indirect stream of tap water until no colour appeared in the effluent. It was re-decolorized with acid alcohol for 60 seconds or until no more red colour ran from the smear. Precaution was taken with decolorize until the fluid washing off the slide ran clear. The slide was then rinsed with tap water. The slide was then flooded with counterstain methylene blue for $5 \mathrm{~min}$. The slide was again rinsed with tap water and dried for examination 
under the phase contrast microscope (Olympus CX-31, Japan) under 100x magnification (oil immersion) [14]

Regarding sex distribution among the participants, there were 11 $(8.66 \%)$ females and $116(91.33 \%)$ males in HIV+ group. The distribution of age in HIV+ patients as shown in fig. 1. Out of the total number of 127 HIV+ patients, $19.68 \%$ (25 cases) were infected with Cryptosporidium sp. In addition, the frequency of infection was observed using CD4 counting. In a group number $<200$ CD4, 15 patients $(11.81 \%)$ were infected, in the group with CD4 counts 200 300,8 patients $(6.2 \%)$ were infected and in the group with CD4 counting $>300$ two patient $(1.57 \%)$ had Cryptosporidium spp. Also, the patients for the study were divided into 5 age groups. Their number and out of 25 people infected with Cryptosporidium who were studied, 13 people were suffering from chronic diarrhea (table 1). The results also show that the lower CD 4 counts $<200$ had more possibilities of Cryptosporidium infection and an increase in CD4 counts and consequently, an increase in immune system resistance; the rate of infection decreases (fig. 2).

In this study, the most common parasites seen in patients with diarrhoea were Cryptosporidium sp. (19.68 \%) having both round and oval in shapes with variable size range (3-6 $\mu \mathrm{m})$, which appeared pink against a light-blue background (fig. 3).

Table 1: Comparison of CD4 counting with Cryptosporidium infection with chronic diarrhoea

\begin{tabular}{|c|c|c|c|}
\hline S. No. & CD4 countsc CD4 counts & Diarrhoea & Cryptosporidium infection \\
\hline 1 & 350 & + & + \\
\hline 2 & 154 & + & + \\
\hline 3 & 134 & + & + \\
\hline 4 & 182 & + & + \\
\hline 5 & 255 & + & + \\
\hline 6 & 120 & + & + \\
\hline 7 & 167 & + & + \\
\hline 8 & 136 & + & + \\
\hline 9 & 281 & + & + \\
\hline 10 & 221 & + & + \\
\hline 11 & 167 & + & + \\
\hline 12 & 283 & + & + \\
\hline 13 & 117 & + & + \\
\hline 14 & 189 & + & + \\
\hline 15 & 209 & + & + \\
\hline
\end{tabular}

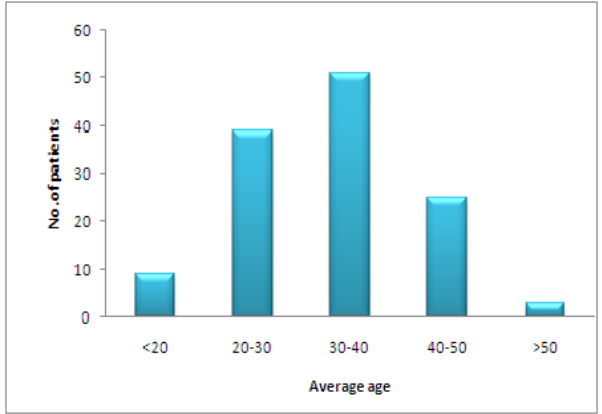

Fig. 1: Comparison age distribution with infected people in HIV+patients admitted to the ART centre silchar medical college and hospital, assam 2013-2014

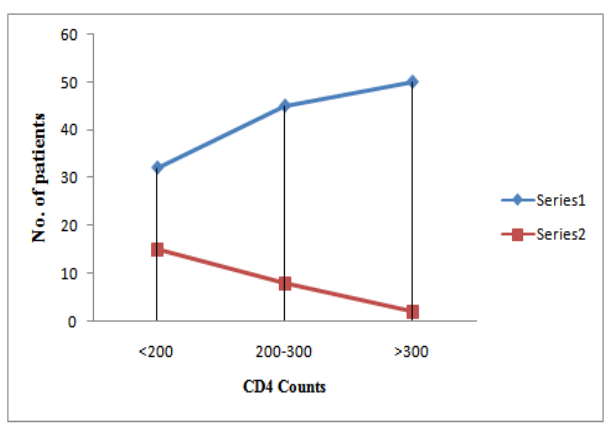

Fig. 2: Comparison infected people with CD4 counts Cryptosporidium in HIV+Patients admitted to the ART center silchar medical college and hospital, assam 2013-2014

Series 1: Total no of sample

Series 2: Cryptosporidium infection

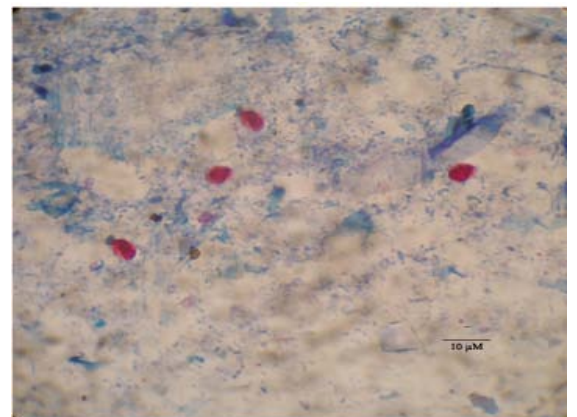

Fig. 3: Cryptosporidium oocysts were seen under phase contrast microscope (Olympus CX-31, Japan)

Cryptosporidium infections are the commonest and major cause of morbidity and mortality in HIV-positive cases in the worldwide. These organisms usually cause a self-limiting illness in immunecompromised patients; they can cause life-threatening, severe diarrhea [15]. HIV infection is a major threat to public health in India. It would be advisable to recognize that cryptosporidiosis can present with just chronic weight loss. Additionally, laboratory testing for Cryptosporidium in HIV-infected patients is highly recommended in order to have a better understanding of the epidemiology and management [16]. Modified Z-N staining method was used for identification of the Cryptosporidium oocysts in the present study; the study was conducted to compare the positivity of acid-fast staining technique in HIV positive diarrheal stool samples ( $\mathrm{n}=127)$. Cryptosporidium infection was detected in 25/127 (19.68\%) of the diarrheal stool samples from HIV-positive patients in the present study, while the same prevalence rate of Cryptosporidium infection was found Gupta et al. [17] and Amatya et al. [18].

Symptomatic cryptosporidiosis was first noted in turkeys in 1955 [19]. During the 1970s, Cryptosporidium infections were reported to cause neonatal diarrhoea in calves. The first human cases of cryptosporidiosis in humans were recorded in the 1970s, one with a young girl with enterocolitis [20]. The other records were in AIDS 
patient with the spread of AIDS in the 1980s [21] and 1990s more and more cases of cryptosporidiosis were diagnosed [22]. Apart from the acute symptoms, there are potential long-term consequences of Cryptosporidium infections. Infection at a young age can lead to impaired development, growth and possible long-term cognitive deficits, especially among children in the developing world [23]

It must be noted that these studies can be difficult to conduct because of the stigmatizing nature of the illness. During the course of this study, many problems were encountered because patients did not return for scheduled visits to the hospital and when home visits were made, it was discovered that patients had moved or provided incorrect addresses. Such problems have also been encountered by other investigators, who have shown that approximately 70 percent of individual's returns with the results of HIV-related tests, and less than 50 percent participated in further studies [24, 25].

The modified Z-N staining technique was less sensitive for the detection of Cryptosporidium in the study population; however, it has the advantage of being the only technique that only indicates active infections, unlike the ELISA and PCR techniques which may not distinguish between active and non-active infections. Moreover, less expertise the Z-N staining technique, although the use of this technique means that several cases of cryptosporidiosis will go undiagnosed as it less sensitive.

The majority of patients suffering from cryptosporidiosis were immune-compromised. Modified acid-fast staining is an economical and easily applicable method in the detection of Cryptosporidium oocysts in faecal samples. This test should be made available routinely as a screening tool of diarrhoeal stool samples. It is not considered at risk to others and that no specific treatment is available for it. It may be rare because it is rarely investigated. It is usually self-limiting, but can be chronic and life threatening in immune-compromised patients. The diagnosis of Cryptosporidium is not difficult and can be achieved by simple conventional staining methods like to modified Z-N staining. However, depending on the facilities available, Z-N staining is easy to use in the detection of Cryptosporidium oocysts. Routine testing of stool will help to explore the severity of the infection and help to reduce morbidity and mortality associated with Cryptosporidium infection.

\section{CONFLICT OF INTERESTS}

\section{Declared none}

\section{REFERENCES}

1. Das P, Roy SS, Mitra Dhar K, Dutta P, Bhattacharya MK, Sen A, et al. Molecular characterization of Cryptosporidium spp. from children in Kolkata, India. J Clin Microbiol 2006;44:246-9.

2. Ajjampur SS, Asirvatham JR, Muthusamy D, Gladstone BP, Abraham OC, Mathai D, et al. Clinical features and risk factors associated with cryptosporidiosis in HIV-infected adults in India. Indian J Med Res 2007;126:553-7.

3. Hunter PR, Nichols G. Epidemiology and clinical features of Cryptosporidium infection in immune-compromised patients. Clin Microbiol Rev 2002;15:145-54.

4. Manabe YC, Clark DP, Moore RD, Lumadue JA, Dahlman HR, Belitsos PC, et al. Cryptosporidiosis in patients with AIDS: correlates of disease and survival. Clin Infect Dis 1998;27:536-42.

5. Joshi M, Chowdhary AS, Dalal PJ, Maniar JK. Parasitic diarrhoea in patients with AIDS. Natl Med J India 2002;15:72-4.

6. Agarwal A, Ningthouja S, Sharma D, Mohen Y, Singh NB. Cryptosporidium and HIV. J Indian Med Assoc 1998;96:276-7.

7. Anand L, Dhanachand C, Brajachand N. Prevalence and epidemiologic characteristics of opportunistic and nonopportunistic intestinal parasitic infections in HIV-positive patients in Manipur. J Communicable Diseases 1998;30:19-22.
8. Tyzzer EE. A sporozoan found in the peptic glands of the common mouse. Proc Soc Exp Biol Med 1907;5:12-3.

9. Tzipori S, Ward H. Cryptosporidiosis: biology, pathogenesis, and disease. Microbes Infect 2002;4:1047-58.

10. Harp JA. Cryptosporidium and host resistance: historical perspective and some novel approaches. Anim Health Res Rev 2003;4:53-62.

11. Houpt ER, Bushen OY, Sam NE, Kohli A, Asgharpour A, Ng CT, et al. Short report: asymptomatic Cryptosporidium hominis infection among human immunodeficiency virus-infected patients in Tanzania. Am J Trop Med Hyg 2005;73:520-2.

12. Bertrand TJ. Diffusion of Innovations and HIV/AIDS. J Health Communication 2004;9:113-21.

13. Flayer R. Cryptosporidium: a water-borne zoonotic parasite. Vet Parasitol 2004;126:37-56.

14. Potters I, Esbroeck MV. Negative staining technique of Heine for the detection of Cryptosporidium spp: a fast and simple screening technique. Open Parasitol J 2010;4:1-4.

15. Narayana Shrihari, Kumudini TS, Mariraj J, Krishna S. The prevalence of Cryptosporidiosis in HIV seropositive individuals and detection of Cryptosporidium oocysts by modified ziehl neelsen staining, Giemsa staining and Sheather sugar floatation technique in a tertiary care hospital. J Pharm Biomed Sci 2011;12 :1-4.

16. Masarat S, Ahmed F, Christi M, Hanid S, Ahmad Sofi B. Prevalence of Cryptosporidium species among HIV-positive asymptomatic and symptomatic immigrant population in Kashmir, India. Iran J Microbiol 2012;4:34-8.

17. Gupta K, Bala M, Deb M, Muralidhar S, Sharma DK. Prevalence of intestinal parasitic infections in HIV-infected individuals and their relationship with immune status. Indian J Med Microbiol 2013;31:161-5

18. Amatya R, Shrestha R, Poudyal N, Bhandari S. Opportunistic intestinal parasites and CD4 count in HIV infected people. J Pathol Nepal 2011;1:118-21.

19. Nichols G, Chalmers R, Lake L, Sopwith W, Regan M, Hunter P, Grenfell P, et al. Cryptosporidiosis: a report on the surveillance and epidemiology of Cryptosporidium infection in England and Wales. In: Cryptosporidium Epidemiology, Final Version; 2006. Available from: http://dwi.defra.gov.uk/research/completed-research/ reports/DWI70_2_201.pdf. [Last accessed on 14 Sep 2012].

20. Nime FA, Burek JD, Page DL, Holscher MA, Yardley JH. Acute enterocolitis in a human being infected with the protozoan Cryptosporidium. Gastroenterology 1976;70:592-8.

21. Meisel JL, Perera DR, Meligro C, Rubin CE. Overwhelming watery diarrhoea associated with a Cryptosporidium in an immunesuppressed patient. Gastroenterology 1976;70:1156-60.

22. Dillingham RA, Lima AA, Gruerrant RL. Cryptosporidiosis: epidemiology and impact. Microb Infect 2002;4:1059-66.

23. Amatya R, Shrestha R, Poudyal N, Bhandari S. Opportunistic intestinal parasites and CD4 count in HIV infected people. J Pathol Nepal 2011;1:118-21.

24. Perez F, Mukotekwa T, Miller A, Orne-Gliemann J, Glenshaw M, Chitsike I, et al. Implmenting a rural programme of prevention of mother-to-child transmission of HIV in zimbabwe: first 18 mo of experience. Trop Med Int Health 2004;9:774-83.

25. Kilewo C, Massake A, Lyamuya E, Semali I, Kalokala F, Ulrassa $\mathrm{E}$, et al. HIV counselling and testing of pregnant women in sub Saharan Africa: experiences from a study on prevention of mother-to-child transmission in dar-es-salaam, Tanzania. J Acquired Immune Defic Syndr 2001;15:458-62.

\section{How to cite this article}

- Gulzar Hussain, Shubhadeep Roychoudhury, Baby Singha. Cryptosporidiosis: an emerging enteric disease in hiv infected patients, barak valley, Assam, India. Int J Pharm Pharm Sci 2016;8(9):304-306. 